\title{
Impulse response shortening in TDOA algorithm for underwater acoustic localization
}

\author{
Shingo Yoshizawa \\ Kitami Institute of Technology, Kitami, Hokkaido 0908507, Japan \\ Received: May 25, 2021. Received: October 12, 2021. Accepted: October 24, 2021. Published: November 3, 2021.
}

\begin{abstract}
This paper presents a new idea of impulse response shortening in time difference of arrival (TDOA) measurement algorithm for underwater acoustic localization. Due to strong multipath interference caused by acoustic wave reflection, the pseudo-peaks occur when calculating a correlation function. Their pseudo-peaks disturbs the detection of a correct time position and leads to large TDOA measurement errors. In our previous paper, we presented the impulse response based GCC-PHAT (IRGCC-PHAT), which computes a time difference by taking a cross-correlation between two impulse responses. As the further improvement, we study the effects of impulse response shortening that removes the pseudo-peaks. The effectiveness of impulse response shortening is explained by observing acoustic wave paths on an acoustic propagation simulator and verified by the simulation results.
\end{abstract}

Keywords-direction of arrival, multipath interference, underwater acoustic localization, time difference of arrival

\section{INTRODUCTION}

$\mathrm{U}$ NDERWATER acoustic localization plays an important role in knowing the positions of underwater vehicles such as remotely operated vehicles (ROVs) and autonomous underwater vehicles (AUVs). Since a radio wave of global positioning system (GPS) is difficult to propagate underwater, an acoustic signal is used to transmit and receive a long-range signal.

The techniques of underwater acoustic localization apply to various types of underwater positioning systems named as long baseline (LBL), short baseline (SBL), and ultra-short baseline (USBL) systems. In USBL systems, a small array of hydrophones is mounted on a receiver unit and the direction of arrival (DOA) of a sound source is estimated using the received signals.

The DOA estimation is categorized in two methods. One is beamforming that generates a power profile various incoming angles with combing received signals [1]. The other method is time difference of arrival (TDOA) measurement that calculates a cross correlation function between two received signals and detects a time difference [2]. Although the DOA estimation in acoustic waves is mainly discussed in the fields of room acoustics and speech and audio processing, the recent literatures regarding to underwater acoustic localization are found in both beamforming [3][4] and TDOA measurement [5]-[7].

This paper focuses on TDOA measurement that can estimate a DOA with a simple array having two receiver hydrophones. The TDOA measurement algorithm computes an arrival time difference between received signals by a correlation function [8][9]. In the related works, generalized cross-correlation with phase transform (GCC-PHAT) and matched filter (MF) are used in [5] and [6], respectively. GCCPHAT calculates a cross-correlation function in frequency domain and detects a time difference by detecting the peak position. In underwater acoustic localization, an artificially generated signal such as a pseudo noise (PN) code sequence can be utilized as a sound source. MF takes the cross-correlation between the received signal and the reference signal given by a replica of the transmitted signal. MF is superior to GCC-PHAT with respect to noise tolerance.

TDOA measurement is strongly influenced by the reflection of sound waves. In underwater acoustics, there are many reflected waves caused by the reflection on water surface, bottom, and obstacles. It is known as the phenomenon of multipath interference and generates the pseudo-peaks in the correlation function. In our previous work, we presented impulse response based GCC-PHAT (IR-GCC-PHAT) to cope with multipath interference [7]. IR-GCC-PHAT computes a time difference by taking a cross-correlation between two impulse responses. We demonstrated that IR-GCC-PHAT shows higher position accuracy than GCC-PHAT and MF in the evaluation of simulation and experiment.

As a further improvement, we propose a new idea of impulse response shortening for TDOA measurement in order to mitigate the influence of multipath interference. Impulse response shortening has been used in other areas such as decreasing a reverberation time in room acoustics [10] and reducing inter-symbol-interference (ISI) in wired communication [11]. In these studies, the impulse response shortening is implemented by a digital filter and convolution between a received signal and filter coefficients is required. On the other hand, impulse response shortening in TDOA measurement can be achieved by only masking a part of the 
impulse response data with zero values.

The effectiveness of impulse response shortening is explained by analyzing acoustic wave reflection paths on an acoustic propagation simulator. We evaluate the position accuracy by comparing the proposed and conventional methods in simulation.

\section{TDOA MEASUREMENT ALGORITHM}

Most of the explanations of TDOA algorithms have been made in our previous work [7]. We describe some of them again for readability.

\section{A. Signal Model}

Two received signals $y_{1}(k)$ and $y_{2}(k)$ can be modeled by using a transmitted signal $x(k)$ and impulse responses $h_{1}(k)$ and $h_{2}(k)$ that express a propagation path from a transmitter to a receiver as

$$
\begin{aligned}
& y_{1}(k)=h_{1}(k) * x(k)+n_{1}(k) \\
& y_{2}(k)=h_{2}(k) * x(k)+n_{2}(k),
\end{aligned}
$$

where $k$ indicates a discrete time index and $*$ shows a convolution operation. $n_{1}(k)$ and $n_{2}(k)$ are noise component uncorrelated with the transmitted signal. The received signals can be expressed in frequency domain as

$$
\begin{aligned}
& Y_{1}(l)=\operatorname{DFT}_{N}\left[y_{1}(k)\right]=H_{1}(l) X(l)+N_{1}(l) \\
& Y_{2}(l)=\operatorname{DFT}_{N}\left[y_{2}(k)\right]=H_{2}(l) X(l)+N_{2}(l) .
\end{aligned}
$$

$\mathrm{DFT}_{N}[\cdot]$ indicates the discrete Fourier transform for $N$ samples and $l$ denotes a discrete frequency index.

When the arrival time difference is obtained by the TDOA algorithm, an angle of DOA is computed as

$$
\theta=\arcsin \left(\frac{c \tau}{d}\right)
$$

where the arrival time difference for the two received signals is expressed by $\tau$. An array space between receiver elements is given by $d$ and a sound velocity is given by $c$.

\section{B. GCC-PHAT}

GCC-PHAT correlation function is given by the following equation:

$$
\Phi_{\mathrm{GCC}-\mathrm{PHAT}}(k)=\operatorname{IDFT}_{N}\left[\frac{Y_{1}(l) Y_{2}^{*}(l)}{\left|Y_{1}(l) Y_{2}^{*}(l)\right|}\right] .
$$

The time difference is detected by the highest peak detection as

$$
\tau_{\mathrm{GCC}-\mathrm{PHAT}}=\underset{k}{\operatorname{argmax}} \Phi_{\mathrm{GCC}-\mathrm{PHAT}}(k) .
$$

C. $M F$

The cross spectrum calculation of MF is computed as

$$
\begin{aligned}
& \Phi_{\mathrm{MF} 1}(k)=\operatorname{IDFT}_{N}\left[Y_{1}(l) X^{*}(l)\right] \\
& \Phi_{\mathrm{MF} 2}(k)=\operatorname{IDFT}_{N}\left[Y_{2}(l) X^{*}(l)\right]
\end{aligned}
$$

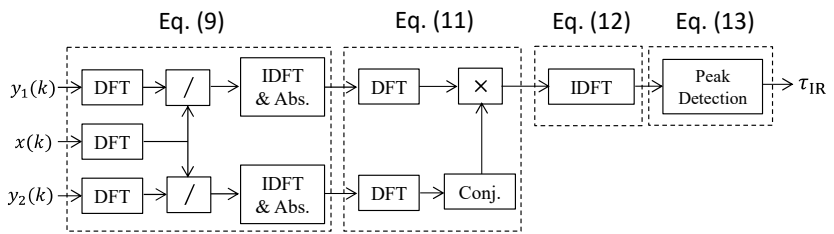

Fig. 1 Procedure of IR-GCC-PHAT algorithm.

The time difference is detected from the two time positions with the highest peaks, expressed as

$$
\tau_{\mathrm{MF}}=\underset{k}{\operatorname{argmax}}\left|\Phi_{\mathrm{MF} 1}(k)\right|-\underset{k}{\operatorname{argmax}}\left|\Phi_{\mathrm{MF} 2}(k)\right| .
$$

\section{IR-GCC-PHAT}

IR-GCC-PHAT directly computes the two impulse responses by the frequency-domain division that is expressed as

$$
\begin{aligned}
h_{1}^{\prime}(k) & =\operatorname{IDFT}_{N}\left[Y_{1}(l) / X(l)\right] \\
& =\operatorname{IDFT}_{N}\left[H_{1}(l)+N_{1}(l) / X(l)\right] \\
h_{2}^{\prime}(k) & =\operatorname{IDFT}_{N}\left[Y_{2}(l) / X(l)\right] \\
& =\operatorname{IDFT}_{N}\left[H_{2}(l)+N_{2}(l) / X(l)\right] .
\end{aligned}
$$

The term of $N_{1}(l) / X(l)$ is transformed as

$$
\frac{N_{1}(l)}{X(l)}=\frac{N_{1}(l) X^{*}(l)}{|X(l)|^{2}}
$$

The time difference is detected by the cross correlation after taking absolute values for the two impulse responses:

$$
\begin{aligned}
G_{1}(l) & =\operatorname{DFT}_{N}\left[\left|h_{1}^{\prime}(k)\right|\right] \\
G_{2}(l) & =\operatorname{DFT}_{N}\left[\left|h_{2}^{\prime}(k)\right|\right] \\
\Phi_{\mathrm{IR}}(k) & =\operatorname{IDFT}_{N}\left[\frac{G_{1}(l) G_{2}^{*}(l)}{\left|G_{1}(l) G_{2}^{*}(l)\right|}\right] \\
\tau_{\mathrm{IR}} & =\underset{k}{\operatorname{argmax}} \Phi_{\mathrm{IR}}(k) .
\end{aligned}
$$

The whole procedure of IR-GCC-PHAT is illustrated in Fig. 1. It corresponds to the combination of MF and GCC-PHAT.

\section{Proposed Algorithm}

\section{A. SIR-GCC-PHAT}

We name the proposed algorithm as shortened impulse response based GCC-PHAT (SIR-GCC-PHAT). First, the time position of the highest peak in the impulse response of $h_{1}^{\prime}(k)$ is detected as

$$
s_{\max }=\underset{k}{\operatorname{argmax}}\left|h_{1}^{\prime}(k)\right| \text {. }
$$

Based on the peak position, impulse response shortening is carried out by the following equations:

$$
\begin{gathered}
\left|h_{1}^{\prime \prime}(k)\right|=\left\{\begin{array}{cc}
\left|h_{1}^{\prime}(k)\right| & \left(k \leq s_{\max }+s_{\mathrm{I}}\right) \\
0 & \left(k>s_{\max }+s_{\mathrm{I}}\right)
\end{array}\right. \\
\left|h_{2}^{\prime \prime}(k)\right|=\left\{\begin{array}{cc}
\left|h_{2}^{\prime}(k)\right| & \left(k \leq s_{\max }+s_{\mathrm{I}}\right) \\
0 & \left(k>s_{\max }+s_{\mathrm{I}}\right),
\end{array}\right.
\end{gathered}
$$


where $s_{\max }+s_{\mathrm{I}}$ indicates the length of the shortened impulse response. The computations taking the cross correlation are same as (11) to (13). The determination of $s_{\mathrm{I}}$ is explained in section V.C.

\section{B. Comparison of TDOA measurement algorithms}

Figure 2 shows an example of acoustic paths for the direct and reflected waves from the sound source (TX) to the receiver elements (RX1 and RX2). Although there are a lot of reflected waves, only several acoustic paths are illustrated. For the impulse responses of $\left|h_{1}(k)\right|$ and $\left|h_{2}(k)\right|$, the magnitudes of peaks depend on the path length and the reflection ratio on the boundary surface.

When the path length of one reflected wave is almost the same as that of and the direct wave, the magnitude of the combined peak is comparable to the peak of the direct wave. This phenomenon is derived from the specific condition that the reflection ratio of water surface is almost 1 . As discussed in (9), we should note the uncertainty of impulse response estimation that is affected by noise components. It sometimes induces the phenomenon that the magnitude relationship between the direct and the reflected wave is reversed.

In computing the correlation function of TDOA algorithms, the pseudo peaks disrupt the correct detection of the arrival time difference. First, we compare MF and IR-GCC-PHAT algorithms in the peak detection. Figure 3 shows the detection of the arrival time difference when the pseudo peaks occur. The true time difference is given by $\tau^{\text {true }}$ in the figure.

In the MF algorithm, the time difference is detected by the two time positions with the highest peaks. Although the targeted peaks are $\mathrm{P}_{1}$ and $\mathrm{P}_{1}^{\prime}$, the peaks of $\mathrm{P}_{1}$ and $\mathrm{P}_{2}^{\prime}$ are wrongly detected in this case. These peaks cannot detect the correct time difference $\left(\tau_{\mathrm{MF}} \neq \tau^{\text {true }}\right)$. IR-GCC-PHAT treats the peaks derived from the direct and reflected waves as one group. When comparing the time positions of $\mathrm{P}_{1}$ to $\mathrm{P}_{2}$ and $\mathrm{P}_{1}^{\prime}$ to $\mathrm{P}_{2}^{\prime}$, the time difference of their groups is near to the true time difference. The cross correlation taking their impulse responses emphasizes this group time difference $\left(\tau_{\mathrm{IR}} \approx \tau^{\text {true }}\right)$.

We compare IR-GCC-PHAT with SIR-GCC-PHAT in the further reflected condition, as shown in Fig. 4. The peaks of the reflected waves that arrive from the different direction are far from that of the direct wave at the time position. These peaks raise the noise floor in the cross-correlation function. IR-GCCPHAT occasionally mistakes the time difference detection $\left(\tau_{\mathrm{IR}} \neq \tau^{\text {true }}\right)$.

SIR-GCC-PHAT shortens the impulse responses by replacing a part of the impulse responses with zero values. It eliminates the unnecessary pseudo peaks from the different direction and helps to decrease the noise floor. SIR-GCCPHAT can detect the correct time difference $\left(\tau_{\mathrm{SIR}} \approx \tau^{\text {true }}\right)$. This mechanism is explained in Section V.

SIR-GCC-PHAT is also effective in noisy environments. The noise component appears as numerous small peaks in the impulse responses. These peaks can be eliminated by the impulse response shortening.
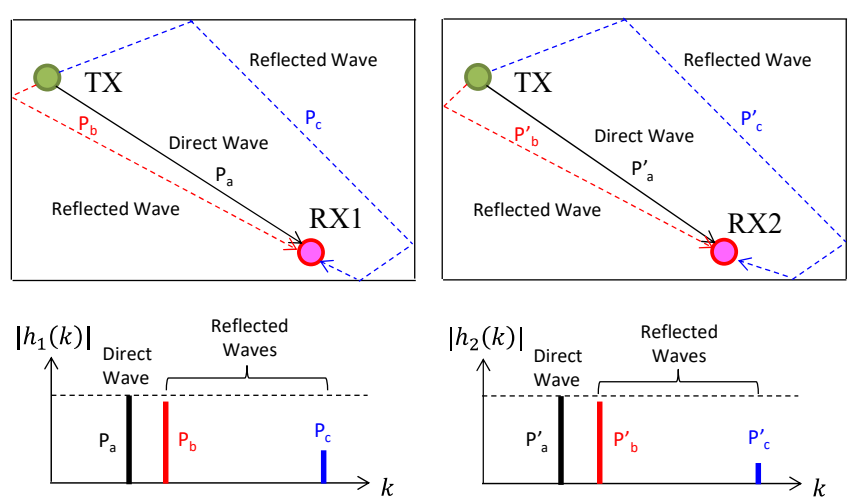

Fig. 2 Acoustic paths for direct and reflected waves.

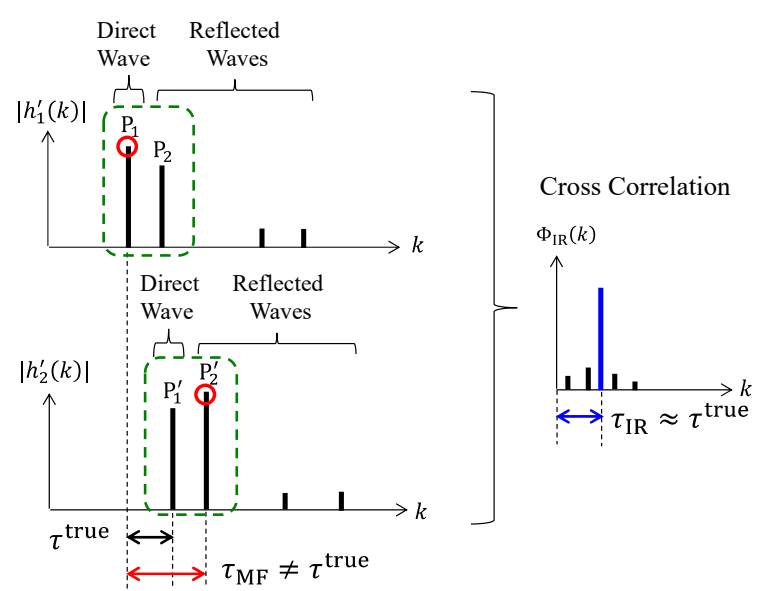

Fig. 3 Comparison of MF and IR-GCC-PHAT algorithms.

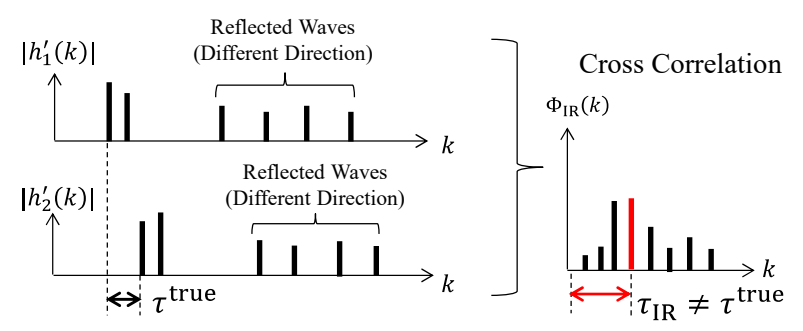

(a) IR-GCC-PHAT

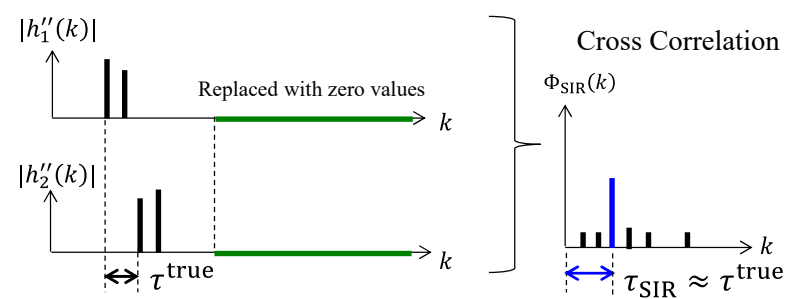

(b) SIR-GCC-PHAT

Fig. 4 Comparison of IR-GCC-PHAT and SIR-GCC-PHAT algorithms. 


\section{Evaluation}

\section{A. Position Estimation}

The comparison of the TDOA algorithms is performed by and the following simple three-dimensional position estimation. When the coordinates of one of the receiver elements are represented by $\boldsymbol{p}_{\mathrm{r}}=\left[x_{\mathrm{r}}, y_{\mathrm{r}}, z_{\mathrm{r}}\right]$, those of the transmitter $\boldsymbol{p}_{\mathrm{t}}=$ $\left[x_{\mathrm{t}}, y_{\mathrm{t}}, z_{\mathrm{t}}\right]$ are computed by the estimated angle $\theta$ :

$$
\begin{aligned}
x_{\mathrm{t}} & =x_{\mathrm{r}}+D \cos \theta \\
y_{\mathrm{t}} & =y_{\mathrm{r}}+D \cos \phi \\
z_{\mathrm{t}} & =z_{\mathrm{r}}-V \\
\cos \phi & =\sqrt{1-\cos ^{2} \theta-\left(\frac{V}{D}\right)^{2}},
\end{aligned}
$$

where $D$ indicates the distance between the receiver and the transmitter positions and $V$ is the vertical position difference. We assume that the parameters of $D$ and $V$ are ideally known for simplify in the evaluation. We briefly describe how to measure the distance and the vertical position below.

In the distance measurement, we assume the time synchronization scheme that transmitter and receiver units have the same clock timing. After the transmitter unit starts sending a signal, the receiver unit measures the time delay $T$ as a sound propagates underwater. The distance can be measured by $D=$ cT. In [12], the time synchronization has been achieved by integrating a chip-scale atomic clock (CSAC) into an acoustic modem.

The vertical position can be measured by the depth sensor. The depth sensor converts an underwater pressure value into a position from water surface. In [13], a pressure sensor (e.g., Blue Robotics Bar30) was used in a sensor suite for underwater reconstruction. As long as the depth sensor is connected to the receiver unit by a wired communication cable, the depth information is easily obtained. If not, we should consider how to send the depth information in underwater wireless communication. This solution would be discussed in our future paper.

\section{B. Simulation Conditions}

Table 1 presents the specifications of the transmitted signal and the simulation conditions. The pseudo noise signal generated by the PN code sequence is used as the transmitted signal. The frequency band of the transmitted signal is $12 \mathrm{kHz}$ to $32 \mathrm{kHz}$, given by a flat spectrum with approximately $|X(l)|=1$ within the band. The acoustic field size is $25 \times 15$ $\times 5 \mathrm{~m}$ (length, width, and height), and the reflectance ratios are set to 1 for water surface and 0.8 for water bottom and all surrounding walls.

The locations of the transmitter (TX) and receiver elements (RX1 and RX2) are shown in Fig. 5. TX is moved every $2 \mathrm{~m}$ along the $\mathrm{X}$-axis (2.5 to $22.5 \mathrm{~m}$ ) and the $\mathrm{y}$-axis ( 8 to $12 \mathrm{~m}$ ) and $0.5 \mathrm{~m}$ along the $\mathrm{z}$-axis $(0.5 \mathrm{~m}$ to $4 \mathrm{~m})$. RX1 and RX2 are fixed at $[12.5,0.05,4.5]$ and $[12.2,0.05,4.5]$, respectively. The array space between receiver elements is $0.3 \mathrm{~m}$. The sound velocity is $1480 \mathrm{~m} / \mathrm{s}$.

The signal modeling in (1) is done by using a sound wave propagation simulator [14]. The generated impulse response
Table 1 Specifications of transmitted signal and simulation conditions.

\begin{tabular}{|l|c|}
\hline Sampling frequency & $200 \mathrm{kHz}$ \\
Frequency band & $12 \mathrm{kHz}-32 \mathrm{kHz}$ \\
Measurement time & $250 \mathrm{~ms}$ \\
Transmitted signal & Pseudo-noise $(\mathrm{PN})$ sequence \\
Signal length & $163.8 \mathrm{~ms}$ \\
Number of signal points & 32768 \\
Number of receivers & 2 \\
Receiver array space & $0.3 \mathrm{~m}$ \\
TDOA measurement period & $81.9 \mathrm{~ms}$ \\
DFT size & 16384 \\
Acoustic field & $25 \times 15 \times 5 \mathrm{~m}$ \\
\hline
\end{tabular}

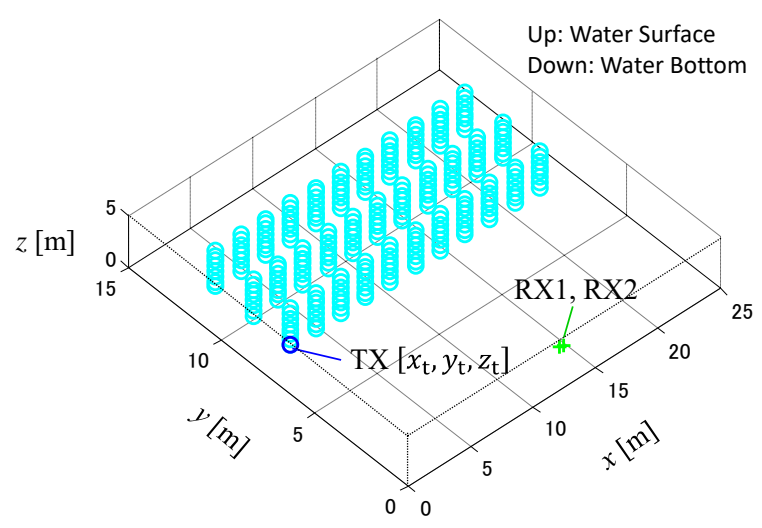

Fig. 5 Locations of transmitter and receiver elements.

depends on the size of acoustic field, the reflectance ratios, and the positions of transmitter and receiver elements. The magnitude of uncorrelated noise is adjusted by a signal-to-noise ratio (SNR). The SNR is given by the ratio of the average signal power of $h_{1}(k) * x(k)$ and the average noise power of $n_{1}(k)$. The amplitude of additive white Gaussian noise (AWGN) is adjusted by the SNR setting value. We evaluate the position errors for the TDOA algorithms where those errors are calculated by the Euclidean distance between true and measured positions. The measured position is computed from (16) where the position of RX1 is used as the coordinates of $\boldsymbol{p}_{\mathrm{r}}$. We apply 326 samples for $s_{\text {I }}$ as for the impulse response shortening in (15). See Section V.C about how to determine the value of $s_{\mathrm{I}}$.

\section{Simulation Results}

The simulation results for a $30 \mathrm{~dB}$ SNR are reported in Table 2. The average position errors for the TDOA algorithms are compared for each TX height. IR-GCC-PHAT is superior to GCC-PHAT and MF in a highly reflective environment as the similar results were previously presented in [7]. SIR-GCCPHAT provides smaller position errors than IR-GCC-PHAT. Average position errors are $3.16 \mathrm{~m}, 2.30 \mathrm{~m}, 0.64 \mathrm{~m}$, and $0.37 \mathrm{~m}$ for GCC-PHAT, MF, IR-GCC-PHAT and SIR-GCC-PHAT, respectively. IR-GCC-PHAT shows the best performance in underwater acoustic localization under reflective environment. 
Table 2 Average position errors for a $30 \mathrm{~dB}$ SNR in meter.

\begin{tabular}{|c|c|c|c|c|}
\hline $\begin{array}{c}\text { TX location } \\
\text { in z-axis }[\mathrm{m}]\end{array}$ & GCC-PHAT & MF & $\begin{array}{c}\text { IR-GCC- } \\
\text { PHAT }\end{array}$ & $\begin{array}{c}\text { SIR-GCC- } \\
\text { PHAT }\end{array}$ \\
\hline 0.5 & 1.47 & 0.42 & 0.54 & 0.37 \\
1.0 & 2.14 & 3.01 & 0.52 & 0.36 \\
1.5 & 2.32 & 2.67 & 0.49 & 0.41 \\
2.0 & 2.74 & 2.89 & 0.51 & 0.31 \\
2.5 & 2.61 & 3.03 & 0.49 & 0.31 \\
3.0 & 4.01 & 2.23 & 0.95 & 0.58 \\
3.5 & 3.25 & 3.14 & 0.89 & 0.30 \\
4.0 & 3.49 & 1.02 & 0.70 & 0.30 \\
\hline Average for all & $\mathbf{3 . 1 6}$ & $\mathbf{2 . 3 0}$ & $\mathbf{0 . 6 4}$ & $\mathbf{0 . 3 7}$ \\
\hline
\end{tabular}

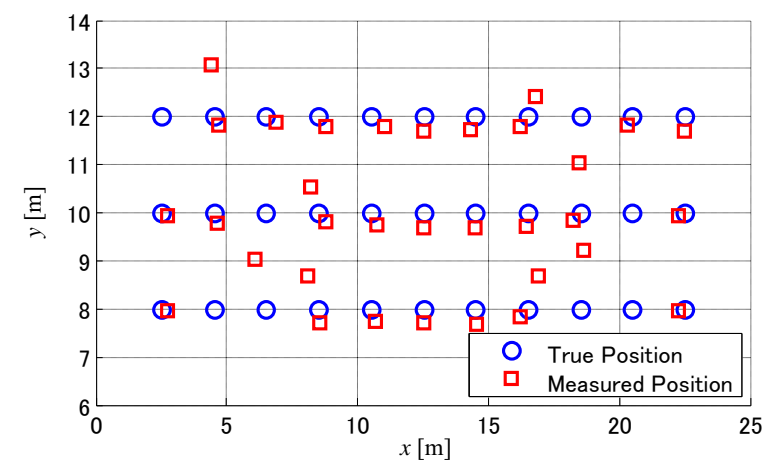

(a) IR-GCC-PHAT

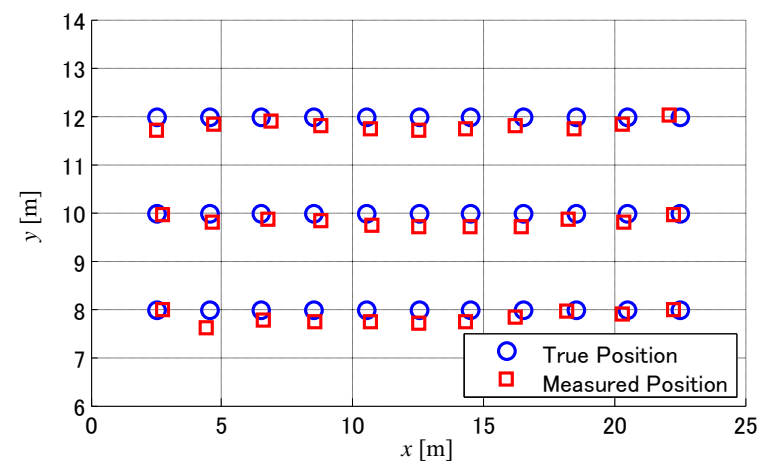

(b) SIR-GCC-PHAT

Fig. 6 Measured positions when TX is located at $4.0 \mathrm{~m}$ in z-axis.

Figure 6 illustrates the measured positions when the TX is located at $4.0 \mathrm{~m}$ in z-axis. IR-GCC-PHAT has large position errors more than $1 \mathrm{~m}$ in some TX locations. On the other hand, SIR-GCC-PHAT keeps high position accuracy for most TX positions. This difference is linked to the results of the average position errors.

The simulation results for the other conditions are summarized in Table 3. Table 3(a) shows the average position errors when decreasing a SNR. The tendency of position accuracy for the TDOA algorithms is the same as Table 2. SIRGCC-PHAT can keep a small position error less than $0.5 \mathrm{~m}$ even in a $-5 \mathrm{~dB}$ SNR.

Table 3(b) gives the average positions for the non-reflective condition, where the reflectance ratios are all zeros. As long as there is no reflection in the acoustic field, MF shows the highest position accuracy. SIR-GCC-PHAT provides higher accuracy
Table 3 Simulation results for other conditions.

(a) Average position errors for other SNR conditions

\begin{tabular}{|c|c|c|c|c|}
\hline SNR [dB] & GCC-PHAT & MF & $\begin{array}{c}\text { IR-GCC- } \\
\text { PHAT }\end{array}$ & $\begin{array}{c}\text { SIR-GCC- } \\
\text { PHAT }\end{array}$ \\
\hline 20 & 2.70 & 2.38 & 0.63 & 0.37 \\
10 & 3.21 & 2.56 & 0.62 & 0.38 \\
0 & 5.32 & 3.02 & 0.62 & 0.31 \\
-5 & 5.75 & 3.21 & 0.76 & 0.49 \\
-10 & 5.73 & 4.40 & 3.92 & 1.99 \\
\hline
\end{tabular}

(b) Average position errors for non-reflective condition

\begin{tabular}{|c|c|c|c|c|}
\hline SNR [dB] & GCC-PHAT & MF & $\begin{array}{c}\text { IR-GCC- } \\
\text { PHAT }\end{array}$ & $\begin{array}{c}\text { SIR-GCC- } \\
\text { PHAT }\end{array}$ \\
\hline 5 & 0.19 & 0.17 & 0.19 & 0.19 \\
0 & 0.25 & 0.17 & 0.19 & 0.19 \\
-5 & 1.88 & 0.17 & 0.19 & 0.19 \\
-10 & 5.73 & 0.17 & 0.20 & 0.19 \\
-15 & 5.76 & 0.17 & 2.92 & 0.30 \\
-20 & 5.76 & 2.16 & 5.80 & 3.53 \\
\hline
\end{tabular}

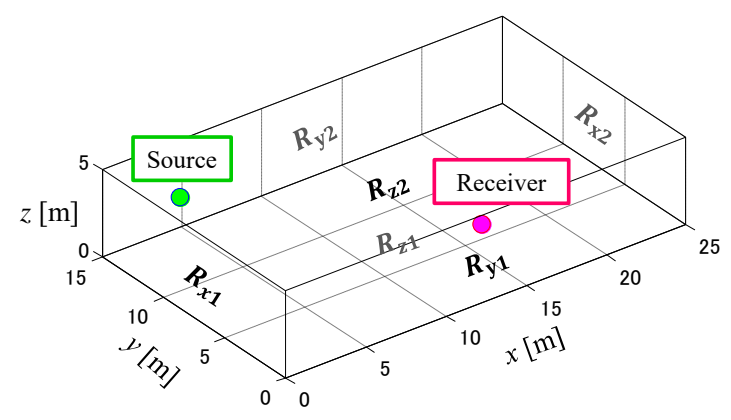

Fig. 7 Settings on sound wave propagation simulator.

than IR-GCC-PHAT in a $-15 \mathrm{~dB}$ SNR. The impulse response shortening can mitigate the influence of noise components when taking the cross correlation function. The noise robustness of SIR-GCC-PHAT is comparable to MF.

\section{AnAlysis of ACOUSTIC REFLECTION PATHS}

\section{A. Sound wave propagation simulator}

We explain the effectiveness of SIR-GCC-PHAT by analyzing acoustic reflection paths on a sound wave propagation simulator. Figure 7 illustrates the settings on the sound wave propagation simulator. The parameters of $\boldsymbol{R}=$ $\left[R_{\mathrm{x} 1}, R_{\mathrm{x} 2}, R_{\mathrm{y} 1}, R_{\mathrm{y} 2}, R_{\mathrm{z} 1}, R_{\mathrm{z} 2}\right]$ give reflectance ratios on the six surfaces. $R_{\mathrm{z} 1}$ and $R_{\mathrm{z} 2}$ are reflectance ratios on the water bottom and surface. The other reflectance ratios on the side walls are expressed as $R_{\mathrm{x} 1}, R_{\mathrm{x} 2}, R_{\mathrm{y} 1}$, and $R_{\mathrm{y} 2}$. Given the coordinates of a sound source and a receiver, acoustic paths and an impulse response from a source to a receiver are analyzed.

Figure 8 shows an example of acoustic paths and impulse responses for the non-reflective condition. The locations of TX, $\mathrm{RX} 1$, and RX2 have been used in the simulation of Section IV. Although the impulse responses in RX1 and RX2 seems to be the same, their arrival times of direct waves are slightly different. The time difference of $0.125 \mathrm{~ms}$ is converted to a DOA angle by (3). 

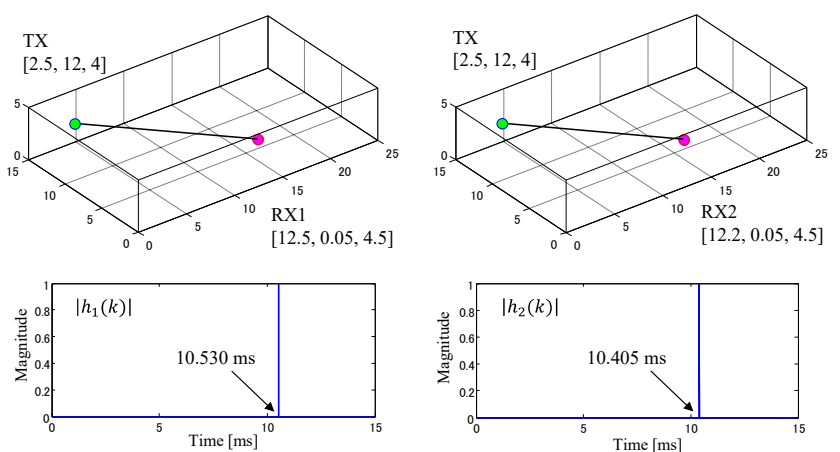

Fig. 8 Example of acoustic paths and impulse responses.

\section{B. TDOA measurement under Acoustic Reflection}

Figure 9 illustrates the acoustic paths and the impulse responses when the reflections on water surface and bottom are valid. The reflection ratio is set to $\boldsymbol{R}=[0,0,0,0,0.8,1]$. The direct wave is observed as the peak of $\mathrm{P}_{1}\left(\mathrm{P}_{1}^{\prime}\right.$ in case of RX2) in the impulse response. The reflected waves are observed as the peaks of $\mathrm{P}_{2}, \mathrm{P}_{3}, \mathrm{P}_{4}, \ldots\left(\mathrm{P}_{2}^{\prime}, \mathrm{P}_{3}^{\prime}, \mathrm{P}_{4}^{\prime}, \ldots\right)$.

Table 4 shows the magnitude and arrive time for the peaks. In the magnitude, these values are normalized by the maximum value. The peak value of $P_{2}$ is almost the same as $P_{1}$. The acoustic path of this reflected wave is highlighted in Fig. 9. Since the path length difference between direct and reflected waves is slight, the peak difference becomes only 0.004. As long as impulse response estimation is ideally done, this peak difference would be correctly detected. However, we should consider the uncertainty related to noise components in (9) and the limitation of time resolution in terms of FFT size and sampling frequency. It is hard to distinguish between $P_{1}$ and $P_{2}$.

In the MF algorithm, the time difference is detected from the two time positions with the highest peaks in RX1 and RX2. When the correct time difference cannot be detected when the magnitude relationship between $\mathrm{P}_{1}$ and $\mathrm{P}_{2}\left(\mathrm{P}_{1}^{\prime}\right.$ and $\left.\mathrm{P}_{2}^{\prime}\right)$ is reversed.

The time differences for the peak combinations between RX1 and RX2 are shown in Table 5. Interestingly, the time difference between $\mathrm{P}_{1}$ and $\mathrm{P}_{1}^{\prime}$ is very similar to that between $\mathrm{P}_{2}$ and $\mathrm{P}_{2}^{\prime}$. It indicates that the DOA angles for the direct waves and the reflected waves are similar in the horizontal direction. IR-GCCPHAT emphasizes only this time difference by taking the cross correlation function for the two impulse responses. We can detect the correct time difference from the highest peak in the cross correlation function (see Fig. 3), independent of the magnitude relationship between the direct and reflected waves.

Figure 10 shows the acoustic paths and the impulse responses under a more reflective condition where the reflection ratio is set to $\boldsymbol{R}=[0.8,0.8,0.8,0.8,0.8,1]$. There are many acoustic paths caused by the reflected waves due to the reflection on the side walls. A lot of peaks are observed in the impulse responses. The reflected waves that arrive at the receiver element after being reflected on the wall have different DOA angles compared to the direct wave. Their peaks does not help to emphasize the correct time difference even for taking the cross
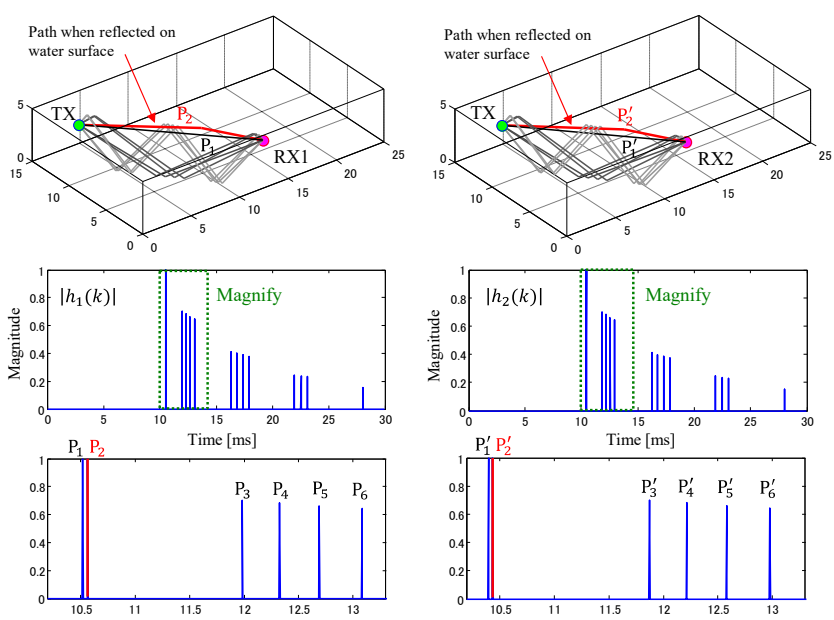

Fig. 9 Acoustic paths and impulse responses for surface and bottom reflection.

Table 4 Magnitude and arrival time for peaks.

(a) Acoustic paths for $\mathrm{RX} 1$

\begin{tabular}{|c|c|c|c|c|c|}
\hline Peak & Magnitude & $\begin{array}{c}\text { Arrival Time } \\
{[\mathrm{ms}]}\end{array}$ & Peak & Magnitude & $\begin{array}{c}\text { Arrival Time } \\
{[\mathrm{ms}]}\end{array}$ \\
\hline $\mathrm{P}_{1}$ & 1.000 & 10.530 & $\mathrm{P}_{1}^{\prime}$ & 1.000 & 10.405 \\
\hline $\mathrm{P}_{2}$ & 0.996 & 10.575 & $\mathrm{P}_{2}^{\prime}$ & 0.996 & 10.445 \\
\hline $\mathrm{P}_{3}$ & 0.702 & 11.990 & $\mathrm{P}_{3}^{\prime}$ & 0.701 & 11.880 \\
\hline $\mathrm{P}_{4}$ & 0.683 & 12.330 & $\mathrm{P}_{4}^{\prime}$ & 0.681 & 12.220 \\
\hline $\mathrm{P}_{5}$ & 0.664 & 12.695 & $\mathrm{P}_{5}^{\prime}$ & 0.661 & 12.585 \\
\hline$P_{6}$ & 0.644 & 13.085 & $\mathrm{P}_{6}^{\prime}$ & 0.641 & 12.980 \\
\hline
\end{tabular}

Table 5 Time differences for peak combinations.

\begin{tabular}{|c|c|}
\hline Combinations & Time Difference $[\mathrm{ms}]$ \\
\hline $\mathrm{P}_{1}$ and $\mathrm{P}_{1}^{\prime}$ & $\mathbf{0 . 1 2 5}$ \\
$\mathrm{P}_{2}$ and $\mathrm{P}_{2}^{\prime}$ & $\mathbf{0 . 1 3 0}$ \\
$\mathrm{P}_{3}$ and $\mathrm{P}_{3}^{\prime}$ & 0.110 \\
$\mathrm{P}_{4}$ and $\mathrm{P}_{4}^{\prime}$ & 0.110 \\
$\mathrm{P}_{5}$ and $\mathrm{P}_{5}^{\prime}$ & 0.110 \\
$\mathrm{P}_{6}$ and $\mathrm{P}_{6}^{\prime}$ & 0.105 \\
\hline
\end{tabular}
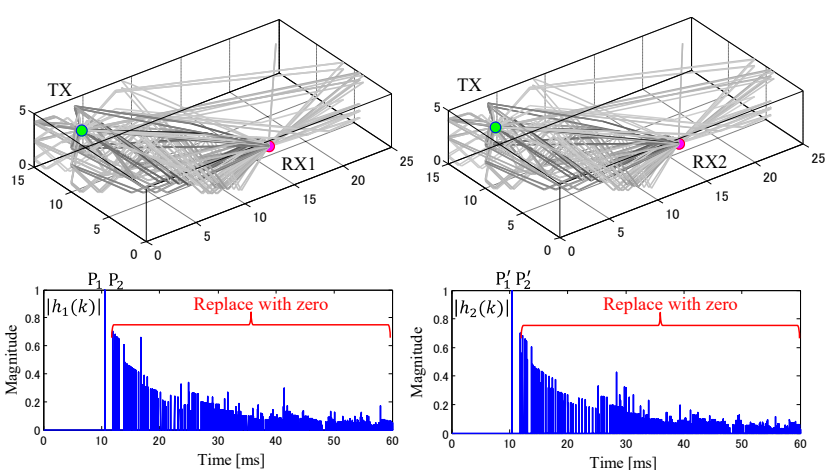

Fig. 10 Acoustic paths and impulse responses for all reflections.

correlation function by IR-GCC-PHAT.

The reasonable way is to eliminate the unnecessary peaks in the impulse responses. It is easily done by replacing a part of 
impulse data with zero values, i.e., the impulse response shortening by SIR-GCC-PHAT. We extract only the peaks of $\mathrm{P}_{1}$ and $\mathrm{P}_{2}\left(\mathrm{P}_{1}^{\prime}\right.$ and $\left.\mathrm{P}_{2}^{\prime}\right)$.

We compare the cross correlation functions between IRGCC-PHAT and SIR-GCC-PHAT in Fig. 11. IR-GCC-PHAT has a high noise floor caused by the reflected waves having individual arrival times. It degrades the sharpness of the cross correlation peak and prevents the detection of the correct time difference. On the other hand, SIR-GCC-PHAT maintains a sharp correlation peak and can find a time difference that is close to the true value.

\section{Determination of length of shortened impulse response}

We explain how to determine an appropriate value of $s_{\mathrm{I}}$ in (15). Since we want to keep the peaks of $P_{1}$ and $P_{2}$ in the impulse response, the arrival time difference between $P_{1}$ and $P_{2}$ becomes a clue. Figure 12 shows the vertical view of the acoustic paths to calculate the maximum path length difference between $\mathrm{P}_{1}$ and $\mathrm{P}_{2}$. Using the parameters related to the TX and RX1 locations and the water depth, the maximum path length difference is computed as

$$
l_{\mathrm{diff}}=2 \sqrt{\left(\frac{l_{\mathrm{max}}}{2}\right)^{2}+h^{2}}-l_{\mathrm{max}}
$$

where $l_{\text {max }}$ denotes the maximum horizontal distance between TX and RX1 and $h$ is the maximum vertical distance between RX1 and water bottom (or water surface). The value of $s_{\mathrm{I}}$ in sample unit can be calculated by

$$
s_{\mathrm{I}}=\frac{l_{\text {diff }}}{c} \cdot f_{s},
$$

where $f_{s}$ is sampling frequency.

In the simulation evaluation, the value of $l_{\max }$ becomes $15.58 \mathrm{~m}$ when the TX is at $[2.5,12]$ as the furthest position and the RX1 is fixed to $[12.5,0.05]$ in the $x-y$ axis. We apply $h=$ $4.5 \mathrm{~m}$ from the vertical distance between the RX1 and the water bottom. The value of $s_{\mathrm{I}}$ becomes 326 samples (1.63 ms).

\section{DISCUSSION}

TDOA measurement is affected by the reflection of sound waves and noise interference. In TDOA measurement algorithms, GCC-PHAT [5] and MF [6] are widely used in underwater acoustic localization. IR-GCC-PHAT has been reported in our previous work [7].

We have proposed SIR-GCC-PHAT based on the impulse response shortening. By analyzing the acoustic paths under reflective environments, we explained that the unnecessary peaks that does not help to emphasize the correct time difference can be removed in the impulse responses. In the simulation evaluation, SIR-GCC-PHAT has shown smaller position errors that IR-GCC-PHAT and others in the highly reflective condition. As reported in Table 2, average position errors are $3.16 \mathrm{~m}, 2.30 \mathrm{~m}, 0.64 \mathrm{~m}$, and $0.37 \mathrm{~m}$ for GCC-PHAT, MF, IR-GCC-PHAT and SIR-GCC-PHAT, respectively.

SIR-GCC-PHAT has provided comparable noise robustness
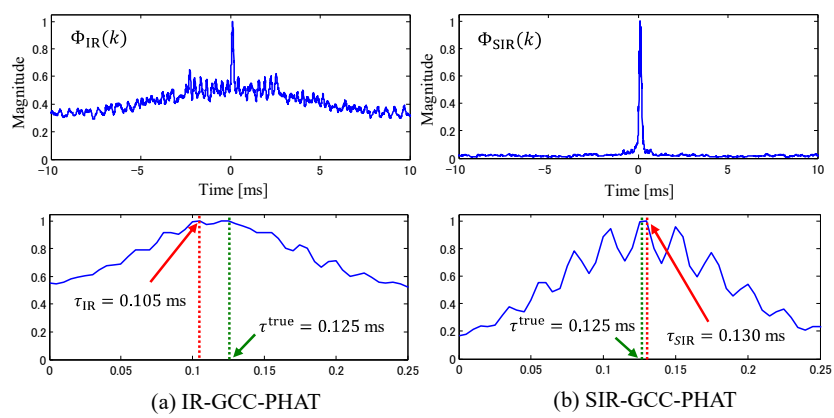

Fig. 11 Comparison of cross correlation functions between IR-GCCPHAT and SIR-GCC-PHAT.

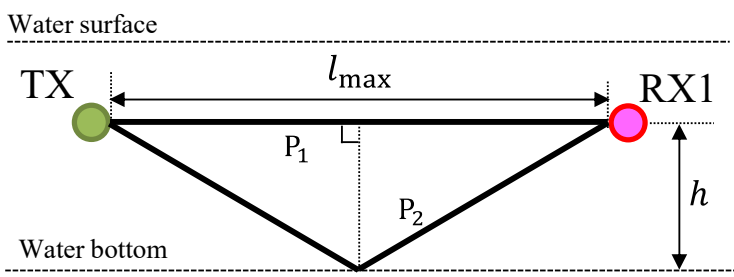

Fig. 12 Vertical view of acoustic paths in direct waves and reflected waves.

to MF. As shown in Table 3(b), MF and SIR-GCC-IR-PHAT can keep smaller position errors less than of $0.5 \mathrm{~m}$ up to a -15 $\mathrm{dB}$ SNR for the non-reflective condition. Although IR-GCCPHAT can keep high position accuracy up to a $-10 \mathrm{~dB}$ SNR, it is slightly inferior to MF and SIR-GCC-PHAT in terms of noise tolerance.

The proposed algorithm is effective when the water depth is shallow and the sound field is surrounded by walls. We assume acoustic positioning of underwater vehicles in a harbor and a dam as a use case.

\section{CONCLUSION}

This paper studied the effect of impulse response shortening in TDOA measurement for underwater acoustic localization. From the acoustic path analysis, we explained that the suppression of unnecessary peaks in impulse responses help to generate a sharp peak with a correct time difference in the cross correlation function. The proposed algorithm showed superior position accuracy compared to other algorithms in the simulation.

In future research, we are planning to present how to apply the proposed algorithm in three-dimensional localization when the depth information is sent in wireless communication.

\section{ACKNOWLEDGMENT}

This work was supported by JSPS KAKENHI Grant Number $20 \mathrm{~K} 04477$.

\section{References}

[1] M. Jian, A. C. Kot, and M. H. Er, "DOA estimation of speech source with microphone arrays," IEEE 
International Symposium on Circuits and Systems (ISCAS), pp. 293-296, May 1998.

[2] J. Chen, J. Benesty, and Y. Huang, "Time delay estimation in room acoustic environments: an overview," EURASIP Journal on Advances in Signal Processing, Vol. 2006, No. 1, pp. 1-19, Dec. 2006.

[3] J. Fischer, C. Doolan, M. Rowan, D. Lamos, J. Seers, O. Vargas, S. Lam, and A. Skvortsov, "Acoustic localization of a buoyancy driven model using a beamforming hydrophone array," Applied Acoustics, Vol. 174, No. 107798, Mar. 2021.

[4] G. Pham, V. Baron, A. Finez, J. I. MARS, and B. Nicolas, "High resolution source localization in underwater acoustics for deep sea mining monitoring," MTS/IEEE OCEANS 2019 - Marseille, pp. 1-7, June 2019.

[5] J. Choi, J. Park, Y. Lee, J. Jung, and H. Choi, "Robust directional angle estimation of underwater acoustic sources using a marine vehicle,” MDPI Sensors, Vol. 18, Issue 9, pp. 1-14, Sep. 2018.

[6] B. Kouzoundjian, F. Beaubois, S. Reboul, J. B. Choquel, and J. Noyer, "A TDOA underwater localization approach for shallow water environment," MTS/IEEE OCEANS 2017 - Aberdeen, pp. 1-4, June 2017.

[7] S. Yoshizawa, "Underwater acoustic localization based on IR-GCC-PHAT in reverberant environments," International Journal of Circuits, Systems and Signal Processing, Vol. 15, pp.164-171, Mar. 2021.

[8] R. Lee, M. Kang, B. Kim, and K. Park, S. Q. Lee and H. Park, "Sound source localization based on GCC-PHAT with diffuseness mask in noisy and reverberant environments," IEEE Access, Vol. 8, pp. 7373-7382, Jan. 2020.

[9] Z. Q. Wang, X. Zhang, and D. Wang, "Robust TDOA estimation based on time frequency masking and deep neural networks," 19th Annual Conference of the International Speech Communication Association (Interspeech 2018), pp. 322-326, Aug. 2018.

[10]A. Mertins, T. Mei and M. Kallinger, "Room impulse response shortening/reshaping with infinity- and p-norm optimization," IEEE Transactions on Audio, Speech, and Language Processing, Vol. 18, No. 2, pp. 249-259, Feb. 2010.

[11] P. J. W. Melsa, R. C. Younce and C. E. Rohrs, "Impulse response shortening for discrete multitone transceivers," IEEE Transactions on Communications, Vol. 44, No. 12, pp. 1662-1672, Dec. 1996.

[12] K. G. Kebkal, O. G. Kebkal, I. Glushko, V. Kebkal et.al, "Underwater acoustic modems with integrated atomic clocks for one-way travel-time underwater vehicle positioning," 4th Underwater Acoustics Conference and Exhibition, pp. 315-323, Sep. 2017.

[13] S. Rahman, N. Karapetyan, A. Q. Li, and I. Rekleitis, "A modular sensor suite for underwater reconstruction," MTS/IEEE OCEANS - Charleston, pp.1-6, Oct. 2018.

[14] D. R. Campbell, K. J. Palomaki, and G. J. Brown, "Roomsim, a MATLAB simulation of shoebox room acoustics for use in teaching and research," Computing and Information Systems Journal, Vol. 9, Issue 3, pp. 1-4, Oct. 2005 .

\section{Creative Commons Attribution License 4.0 (Attribution 4.0 International, CC BY 4.0)}

This article is published under the terms of the Creative Commons Attribution License 4.0

https://creativecommons.org/licenses/by/4.0/deed.en US 